\title{
CARACTERÍSTICAS BIOMÉTRICAS E ÍNDICES DE CONDIÇÃO DA OSTRA-DE-MANGUE Crassostrea rhizophorae (GUILDING, 1828) DO ESTUÁRIO DO RIO IPOJUCA, PE, BRASIL
}

\author{
Lucas L.S. SANTANA ${ }^{1}$; Carmen MEDEIROS2*
}

\begin{abstract}
${ }^{1}$ Programa de Pós-graduação em Oceanografia, Universidade Federal de Pernambuco, Brasil. E-mail: lucaslseixas@gmail.com

${ }^{2}$ Laboratório de Oceanografia Física Estuarina e Costeira, Universidade Federal de Pernambuco, Brasil. Email: cmlimongi@gmail.com

*Autor correspondente
\end{abstract}

\begin{abstract}
RESUMO. Filtradores sésseis e bioacumuladores, as ostras são bons indicadores da saúde dos estuários. Alteração das características morfológicas de suas conchas e da relação peso de sua carne para o volume de sua concha, etc. refletem a qualidade do ambiente onde vivem. O presente trabalho enfoca uma avaliação das condições da ostra-de-mangue Crassostrea rhizophorae no estuário do rio Ipojuca, um dos mais poluídos do país. Para tanto 80 indivíduos de 4 áreas do estuário foram coletados em jan/2019 (pósdesova) e out/2019 (desova), pesados e mensurados. Com base nesses dados foram calculados os índices de espessamento da concha (STI1) e o índice de qualidade da norma francesa AFNOR. Resultados revelaram por um lado, a ocorrência de ostras comercialmente classificáveis como "finas" e mesmo "especiais" em termos de seu desenvolvimento e preenchimento do espaço intervalvar das mesmas. Por outro lado, baixos valores $(<4)$ do índice de monitoramento STI1, forte indicador de contaminação por TBTF, alerta para a necessidade de futuras investigações para confirmar essas suspeitas bem como para investigar a adequabilidade ou não das ostras do Ipojuca ao consumo humano.
\end{abstract}

Palavras-Chave: ostra-de-mangue, Crassostrea rhizophorae, biometria, índices de condição.

\begin{abstract}
As sessile filters and bioaccumulators, oysters are good indicators of the health of estuaries. Changes in the morphological characteristics of its shells and the weight ratio of its flesh to the volume of its shell, etc. reflect the quality of the environment in which they live. The present work focuses on an evaluation of the conditions of the mangrove oyster Crassostrea rhizophorae in the estuary of the Ipojuca River, one of the most polluted in the country. For this purpose, 80 individuals from 4 areas of the Ipojuca river estuary were collected, in jan/2019 (post-spawning) and oct/2019 (spawning), weighted and measured. Based on these data, the shell thickness indices (STI1) and the AFNOR French standard quality index were calculated. Results revealed, on one hand, the occurrence of oysters commercially classifiable as "fine" and even "specials" in terms of their development and filling of their intervalval space. On the other hand, low values $(<4)$ of the STI1 monitoring index, a strong TBT contamination indicator, alerts to the need for future investigations to confirm these suspicions as well as to investigate the suitability or otherwise of Ipojuca oysters for human consumption.
\end{abstract}

Keywords: mangrove oysters, Crassostrea rhizophorae, biometry, condition indexes. 


\section{INTRODUÇÃO}

Os estuários são ambientes aquáticos costeiros de grande importância ecológica por servirem como sítio de reprodução e crescimento e como fonte de alimento e habitat para uma grande variedade de organismos que têm papel importante na cadeia alimentar das águas interiores, estuarinas e oceânicas (CIRM, 1981). São áreas que geralmente apresentam uma boa diversidade e muito produtivas devido à sua capacidade de gerarem e reterem matéria orgânica e nutrientes (Mann, 1982). Por serem propícias ao desenvolvimento de atividades econômicas variadas (navegação, indústria e comércio, urbanização, lazer, pesca e aquicultura, etc.) as áreas estuarinas são palcos de intensa ocupação e vem sofrendo através dos tempos desmatamentos, modificações de suas características geométricas e dos aportes de água doce e marinha que repercutem fortemente no regime salino local, nos processos de circulação e mistura de suas águas, nas trocas de materiais com as áreas costeiras (Miranda et al., 2002) bem como na qualidade de suas águas pelo efeito acumulado dos descartes de efluentes domésticos e industriais ao longo de sua bacia de drenagem. Tudo isso compromete grandemente a prestação de serviços e função ecológica dos estuários e requerem esforços para sua manutenção e recuperação.

Em estuários tropicais, predomina a vegetação de mangue, com uma variedade de nichos ecológicos, abrigando uma fauna diversificada e sendo considerada no Brasil, área de preservação permanente (CÓDIGO FLORESTAL - Lei No 12.651/12). Dentre as espécies de mangue, que ocorrem nos estuários do Nordeste do Brasil, destaca-se o mangue vermelho (Rhizophora mangle L.) que ocorre em amplo gradiente de inundação. As raízes do mangue funcionam como filtros na retenção de sedimentos e nutrientes, incrementando a produtividade local e ao mesmo tempo servindo de refúgio e substrato para diversas espécies dentre as quais a ostra-de-mangue Crassostrea rhizophorae (Guilding, 1828).

A manutenção dos bancos de ostras de sistemas estuarinos é vital para o bom funcionamento e recuperação dos estuários. As ostras sequestram carbono na forma de $\mathrm{CaCO}_{3}$ incorporando-o à matriz de suas conchas (Hargis and Haven, 1999), contribuindo para o balanço global de carbono. As assembleias desses bivalves filtradores contribuem também para a melhoria da qualidade das águas estuarinas reduzindo sua turbidez e eutrofização (Jackson et al., 2001); reduzindo a biomassa microbiana (inclusive de coliformes fecais); estimulando a desnitrificação do sedimento (Newell et al., 2002) e favorecendo o desenvolvimento do microfitobentos. Proveem refúgio e alimento para invertebrados e vertebrados de diferentes níveis tróficos, contribuindo para o incremento da produtividade estuarina e a sobrevivência de muitas espécies de valor ecológico e comercial (Grabowski and Petersen, 2007; Martinez and Rusch, 2014; Neema, 2008).

Por serem organismos sésseis e bioacumuladores, as ostras-de-mangue podem ser utilizadas como indicadores de contaminação, revelando a presença/concentração de alguns 
compostos bionocivos na água. Alterações morfológicas de suas conchas, da espessura da mesma, na relação peso da concha/peso de carne, etc. refletem a qualidade do ambiente onde vivem. Por exemplo, o espessamento e a alteração morfológica da concha para uma forma fora do padrão (mais esférica), podem indicar a presença de TBT (Tributil de Estanho) no ambiente onde vivem esses moluscos.

Neste panorama, o presente estudo enfoca uma avaliação das condições da ostra-demangue Crassostrea rhizophorae no estuário do rio Ipojuca, através de análises morfométricas das mesmas e do cálculo de índice de monitoramento, buscando contribuir com informações que possam subsidiando ações voltadas para a recuperação dos bancos da ostra-de-mangue naquele estuário, e assim contribuir para a recuperação da capacidade produtividade de suas águas.

\section{MATERIAIS E MÉTODOS}

\section{ÁREA DE ESTUDO}

O rio Ipojuca nasce em Arco Verde (Serra Pau D'Arco) e apresenta um curso de cerca de $320 \mathrm{~km}$, desembocando no Atlântico, ao sul do Porto de Suape. Este rio sofreu, dentre os quatro que fluem em direção à bacia de Suape, o maior impacto com a construção do porto: alteração de sua calha, estrangulamento de sua desembocadura e corte do manguezal adjacente ao empreendimento (Braga et al., 1989) e com fortes repercussões para seu regime salino (Lins and Medeiros, 2018). Em seu trecho superior, apresenta regime fluvial intermitente, passando a perene nas proximidades da cidade de Caruaru. Seus principais afluentes são os riachos Liberal, Taquara e do Mel pela margem direita e os riachos do Coutinho, dos Mocós, do Muxoxo e Pata Choca, pela margem esquerda (CONDEPE/FIDEM, 2005).

O estuário do rio Ipojuca experimenta clima tropical quente-úmido, segundo o esquema de classificação climática Köppen (Andrade and Lins, 2001). O regime pluviométrico na região varia de 1500 a $2500 \mathrm{~mm}$.ano ${ }^{-1}$ com uma estação chuvosa de março a agosto e precipitação média mensal de $250 \mathrm{~mm}$; e uma estação de estiagem de setembro a fevereiro, com precipitação média mensal de $70 \mathrm{~mm}$.

Em seu curso oeste-leste em direção ao Oceano Atlântico, o Ipojuca corta importantes sedes municipais como Bezerros, Caruaru, Escada, Chã Grande, Gravatá, Ipojuca, Primavera, São Caetano e Tacaimbó. Boa parte desses Municípios não dispõe de redes para coleta e tratamento adequado de esgoto sanitário, resultando em seu lançamento no rio Ipojuca, in natura ou minimamente tratado. Além da carga orgânica, o Ipojuca recebe também agrotóxicos e fertilizantes carreados pelas chuvas das plantações de cana-de-açúcar e de outras lavouras, efluentes de indústrias açucareira e de curtumes e de indústrias instaladas no Complexo Industrial Portuário de Suape (CIPS). A comunicação restrita com as águas marinhas, através 
Santana and Medeiros, Características biométricas e índices de condição da ostra-de-mangue Crassostrea rhizophorae (Guilding, 1828) do estuário do rio Ipojuca, PE, Brasil.

de uma abertura no recife principal, agrava essa situação ao passo que a presença de manguezais, contribuem para amenizar a situação, devido a seu papel depurador (NeumannLeitão, 1994).

A área geral de estudo (Fig. 1) compreende um trecho de cerca de $2.5 \mathrm{~km}$ do baixo estuário do rio Ipojuca onde ocorre a vegetação de mangue vermelho, Rhizophora mangle e uma maior densidade da ostra-de-mangue Crassostrea rhizophorae.

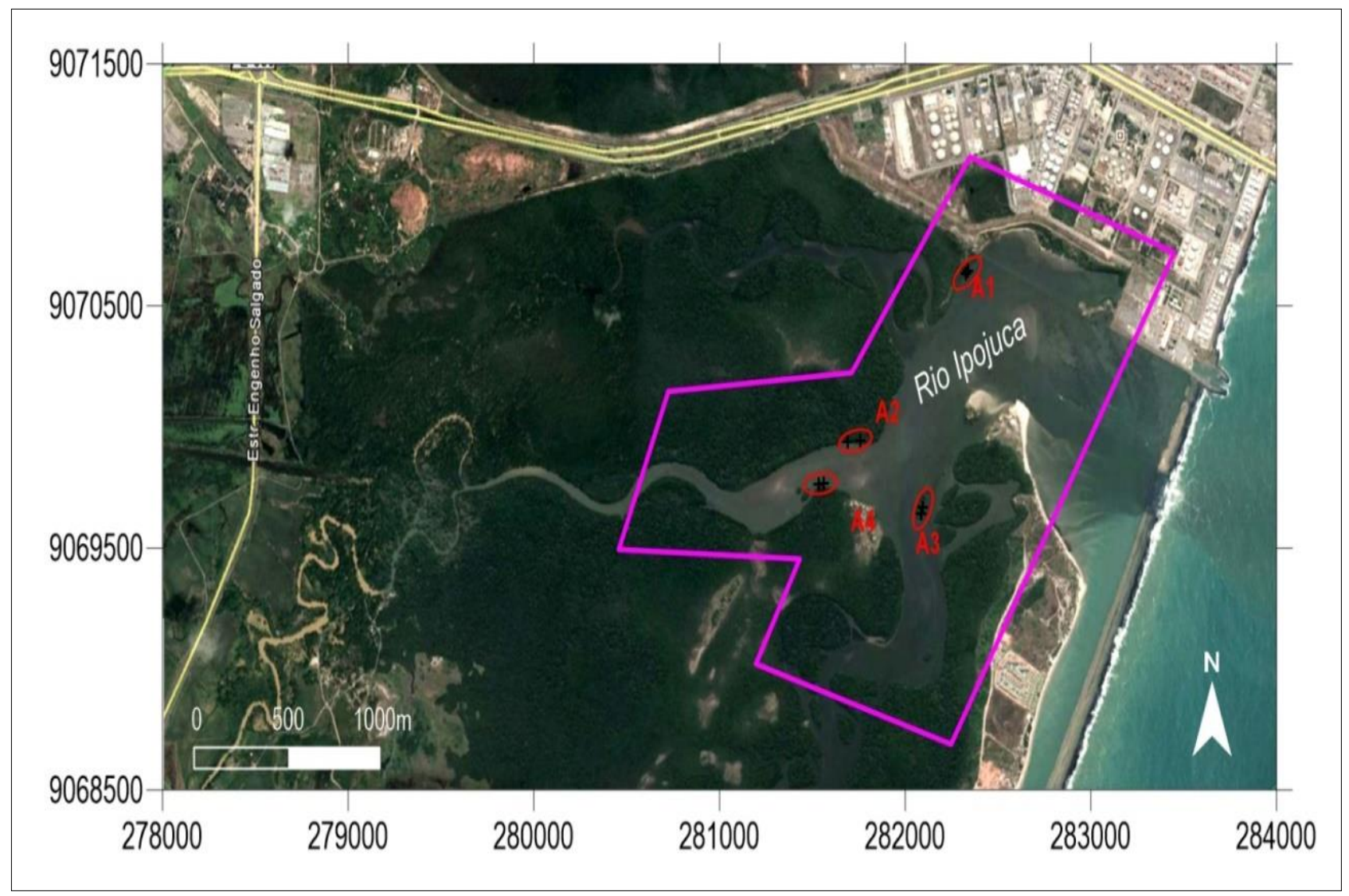

Figura 1. Vista da área geral de estudo, indicada pelo polígono em cor magenta e das áreas amostrais A1-A4 no estuário do rio Ipojuca, Ipojuca-PE.

\section{ÁREAS AMOSTRAIS}

A área geral de estudo foi inicialmente percorrida a bordo de uma embarcação visando mapear as áreas de ocorrência de bancos naturais da ostra-de-mangue e as áreas de amostragem. Quatro áreas foram selecionadas. A Área 1 (A1) está localizada no baixo estuário do Ipojuca e sob maior influência das águas costeiras e marinhas. Aqui as árvores de mangue apresentam raízes bem desenvolvidas, ramificadas e com muitas ostras. A Área 2 (A2) está localizada no médio estuário, na margem esquerda do Ipojuca onde as árvores de mangue apresentam raízes medianamente desenvolvidas e ramificadas, com boa presença de ostras. A Área 3 (A3), localizada no médio estuário, em uma ilhota à confluência com o rio Merepe. Nesta 
área as árvores do mangue-vermelho apresentam raízes medianamente desenvolvidas e ramificadas, baixa densidade e com ostras a elas fixadas. A Área 4 (A4), também no médio estuário, na margem direita do Ipojuca corresponde à estação mais para montante do rio. Nesta última estação as raízes do mangue vermelho apresentam-se medianamente desenvolvidas e ramificadas, com um menor número de ostras. As coordenadas geográficas do centro dessas 4 áreas são sumarizadas na Tab.1 e sua localização indicada na Fig. 1.

Tabela 1. Coordenadas UTM (25L) do centro das áreas experimentais.

\begin{tabular}{ccc}
\hline Área & UTM E & UTM N \\
\hline A1 & 282332 & 9070638 \\
A2 & 281726 & 9069942 \\
A3 & 282092 & 9069653 \\
A4 & 281548 & 9069766 \\
\hline
\end{tabular}

\section{BIOMETRIA}

Vinte exemplares de ostras foram coletados em raízes de cada uma das 4 áreas amostrais em jan/2019 e out/2019 totalizando 160 indivíduos amostrados, 80 em cada período. As coletas foram realizadas durante o estágio de baixa-mar quando as ostras se achavam emersas.

A escolha dos dois períodos amostrais janeiro e outubro esteve ligada ao ciclo reprodutivo das ostras-de-mangue que tem implicações nos índices estudados. Em geral na região, o maior pico de desova ocorre em outubro. Assim, as amostragens em outubro seriam então mais representativas da condição de desova, quando as ostras estariam com suas gônadas mais maduras/repletas, preenchendo melhor a cavidade intervalvar. Já a amostragem em janeiro representaria um período posterior a desova, quando as gônadas das ostras voltariam a um estágio inicial apresentando menor volume e consequentemente menor preenchimento da cavidade intervalvar.

Os exemplares de ostras coletados foram acondicionados em sacos plásticos etiquetados e transportados refrigerados para o LOFEC, Laboratório de Oceanografia Física Estuarina e Costeira para imediata determinação de suas características morfométricas.

Em laboratório, seguindo-se metodologia de Gervis and Sims (1992) e protocolo do Ministério da Ciência e Cultura Português (Projeto Ciência Viva), as ostras foram lavadas e escovadas em água corrente para remoção de sedimentos e algas. Cracas, galerias de poliquetas, sementes, etc. foram removidos com uso de uma chave de fenda, cuidadosamente, para não danificar suas conchas. Em seguida as ostras limpas foram deixadas sobre papel toalha por cerca de 5 minutos, para eliminar o excesso de água. 
Inicialmente foi realizada a biometria das ostras ainda inteiras/fechadas medindo-se: a altura; o comprimento a largura o peso e o volume total de cada uma. Em seguida cada ostra foi aberta inserindo-se um escalpelo entre suas valvas, para separação das mesmas e remoção dos tecidos moles. Estes foram transferidos para uma placa de Petri e deixados por cerca de 1 minuto sobre papel toalha para eliminar excesso de água, medindo-se em seguida seu peso úmido. Mediu-se também o peso e volume da valva direita.

As medidas das alturas (A), comprimentos (C) e larguras ( $L$ ) foram realizadas com uso de um paquímetro com precisão de 0.01 mm, de acordo com Galtsoff (1964) (Fig. 2).
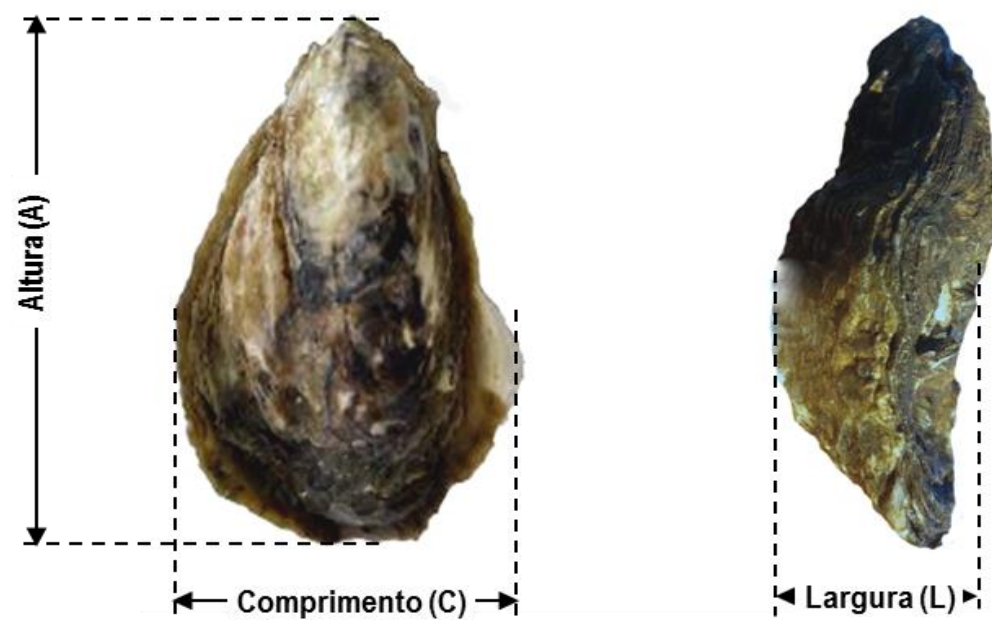

Figura 2. Esquema de medição biométrica das ostras (altura, comprimento e largura) segundo Galtsoff (1964).

A determinação do volume foi obtida por deslocamento do nível de um fluido, empregando-se uma proveta graduada. Na pesagem das ostras e valvas utilizou-se uma balança digital de precisão DIY com resolução de $0.01 \mathrm{~g}$.

Análises de variância e teste de Tukey foram empregados para identificar diferenças significativas $(\mathrm{P}<0.05)$ dos vários parâmetros mensurados entre as áreas e períodos de coleta.

\section{ÍNDICES DE MONITORAÇÃO}

Os valores das dimensões, peso e volume das ostras e de suas valvas e de carne, obtidos a partir das medições biométricas foram utilizados para calcular dos índices de espessamento da concha, Shell Thickness Index, STI1 (Alzieu et al., 1982) e o Índice de qualidade de AFNOR (Soletchnik et al., 2000). Destes, o STI1 proposto para a monitorização do formato das ostras, é o mais amplamente utilizado por já ser conhecida a relação causa-efeito. O mesmo pode ser calculado pela seguinte expressão: 


$$
S T I 1=\frac{a v d}{l v d}
$$

onde, avd é a altura da valva direita em milímetros;

lvd é a largura da valva direita em milímetros.

O STI1 varia de 1 a 10 onde valores de 1 e 2 indicam contaminação muito elevada, de 2 a 4 indicam contaminação elevada; de 5 a 7 indicam contaminação média; de 8 e 9 indicam contaminação baixa e de 10 indicam ausência de contaminação.

O índice de qualidade AFNOR (Soletchnik et al., 2000) exprime em percentagem, a relação entre o peso de carne úmido e o peso total da ostra. Este índice foi desenvolvido segundo a norma francesa NF V45-056 (AFNOR, 1985) para classificar a qualidade das ostras C. gigas produzidas naquele país, mas, posteriormente tem sido aplicado a outros bivalves, também para indicar sua qualidade. Ostras que apresentam um Índice de AFNOR (\%) acima de 9 são consideradas "especiais" por apresentarem um bom preenchimento da cavidade intervalvar; ostras apresentando índices de AFNOR (\%) entre 6,5 e 9,0 são consideradas "finas" e ostras com índice de AFNOR (\%) abaixo de 6,5, são consideradas "sem classificação". Este índice é calculado como:

$$
A F N O R=\frac{p u c}{p o} \times 100
$$

onde, puc é o peso úmido da carne em gramas;

po é o peso total da ostra em gramas.

\section{RESULTADOS}

\section{CARACTERÍSTICAS BIOMÉTRICAS}

Nas Tab. 2 e Fig. 3 são sumarizados os valores da altura, comprimento, largura, peso e volume das ostras amostradas nas 4 áreas e 2 períodos (jan/2019 e out/2019) bem como do peso de carne das mesmas.

Tabela 2. Valores mínimos, máximos e médios \pm desvio padrão (DP) da altura (mm), comprimento $(\mathrm{mm})$, largura $(\mathrm{mm})$, peso $(\mathrm{g})$ e volume $(\mathrm{ml})$ das ostras e do peso de carne $(\mathrm{g})$ 
das mesmas no estuário do rio Ipojuca. Ipojuca-PE. Letras minúsculas distintas denotam diferenças estatísticas significativa entre as médias $(p<0,05)$ das áreas de um mesmo período de coleta obtida pelo teste de Tukey.

\begin{tabular}{|c|c|c|c|c|c|c|c|c|c|}
\hline & & & JAN & 019 & & & OUT & 019 & \\
\hline & & $\mathrm{A} 1$ & $\mathrm{~A} 2$ & A3 & A4 & $\mathrm{A} 1$ & $\mathrm{~A} 2$ & A3 & A4 \\
\hline & Mínimo & 30 & 30 & 34 & 34 & 36 & 19 & 24 & 17 \\
\hline 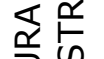 & Máximo & 45 & 45 & 46 & 63 & 49 & 47 & 48 & 44 \\
\hline 战造 & $\begin{array}{l}\text { Média } \\
\pm D P\end{array}$ & $\begin{array}{l}38.40 \\
\pm 3.97\end{array}$ & $\begin{array}{l}37.60 \\
\pm 4.81\end{array}$ & $\begin{array}{l}40.75 \\
\pm 3.40\end{array}$ & $\begin{array}{l}45.40 \\
\pm 6.67\end{array}$ & $\begin{array}{l}41.65 \\
\pm 3.69\end{array}$ & $\begin{array}{l}37.35 \\
\pm 7.02\end{array}$ & $\begin{array}{l}37.20 \\
\pm 5.95\end{array}$ & $\begin{array}{l}35.55 \\
\pm 6.04\end{array}$ \\
\hline & Tukey & a & a & a & $b$ & a & $a b$ & $b$ & $b$ \\
\hline & Mínimo & 20 & 22 & 22 & 19 & 22 & 14 & 17 & 10 \\
\hline 总吕 & Máximo & 34 & 36 & 36 & 32 & 38 & 39 & 38 & 38 \\
\hline 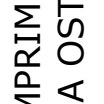 & $\begin{array}{l}\text { Média } \\
\pm D P\end{array}$ & $\begin{array}{l}27.10 \\
\pm 4.54\end{array}$ & $\begin{array}{l}28.45 \\
\pm 4.45\end{array}$ & $\begin{array}{l}30.85 \\
\pm 4.42\end{array}$ & $\begin{array}{l}25.95 \\
\pm 4.87\end{array}$ & $\begin{array}{l}31.55 \\
\pm 5.31\end{array}$ & $\begin{array}{l}26.70 \\
\pm 7.83\end{array}$ & $\begin{array}{l}27.80 \\
\pm 6.53\end{array}$ & $\begin{array}{l}26.15 \\
\pm 6.18\end{array}$ \\
\hline $\mathrm{O}^{\circ}$ & Tukey & $a b$ & $a b$ & $a$ & $b$ & a & bc & $\mathrm{ac}$ & c \\
\hline$\varangle \varangle$ & Mínimo & 12 & 10 & 12 & 9 & 13 & 5 & 7 & 9 \\
\hline 全岕 & Máximo & 29 & 22 & 23 & 23 & 29 & 28 & 26 & 28 \\
\hline $\begin{array}{ll}0 \\
0\end{array}$ & $\begin{array}{l}\text { Média } \\
+\cap D\end{array}$ & $\begin{array}{l}18.45 \\
+\Delta \supset 7\end{array}$ & $\begin{array}{l}16.70 \\
+262\end{array}$ & $\begin{array}{l}18.80 \\
+765\end{array}$ & $\begin{array}{r}14.18 \\
+250\end{array}$ & $\begin{array}{l}18.65 \\
+\Delta \Delta 5\end{array}$ & $\begin{array}{r}16.55 \\
+617\end{array}$ & $\begin{array}{l}16.25 \\
+\Delta 87\end{array}$ & $\begin{array}{r}15.40 \\
+512\end{array}$ \\
\hline & Tukey & $a$ & $a b$ & ac & bd & $a$ & $a$ & $a$ & $a$ \\
\hline & Mínimo & 3.96 & 3.22 & 4.55 & 3.16 & 6.04 & 1.99 & 3.45 & 2.39 \\
\hline$\circ \stackrel{q}{E}$ & Máximo & 12.80 & 14.83 & 12.54 & 18.70 & 18.02 & 15.30 & 14.80 & 12.78 \\
\hline 出 & $\begin{array}{l}\text { Média } \\
\pm D P\end{array}$ & $\begin{array}{c}7.82 \\
\pm 2.59\end{array}$ & $\begin{array}{c}7.21 \\
\pm 2.93\end{array}$ & $\begin{array}{c}8.97 \\
\pm 2.09\end{array}$ & $\begin{array}{c}9.78 \\
\pm 4.64\end{array}$ & $\begin{array}{l}10.17 \\
\pm 3.33\end{array}$ & $\begin{array}{c}8.47 \\
\pm 3.79\end{array}$ & $\begin{array}{c}7.69 \\
\pm 3.08\end{array}$ & $\begin{array}{r}6.66 \\
\pm 3.35\end{array}$ \\
\hline & Tukey & a & a & a & a & a & $a b$ & $a b$ & $b$ \\
\hline & Mínimo & 8 & 7 & 8 & 4 & 13 & 4 & 8 & 10 \\
\hline 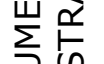 & Máximo & 19 & 27 & 18 & 22 & 22 & 20 & 18 & 18 \\
\hline ○ & $\begin{array}{l}\text { Média } \\
\pm D P\end{array}$ & $\begin{array}{l}12.55 \\
\pm 3.63\end{array}$ & $\begin{array}{l}13.80 \\
\pm 5.20\end{array}$ & $\begin{array}{c}15.3 \\
\pm 2.62\end{array}$ & $\begin{array}{l}12.83 \\
\pm 5.46\end{array}$ & $\begin{array}{l}16.20 \\
\pm 2.53\end{array}$ & $\begin{array}{l}14.15 \\
\pm 4.16\end{array}$ & $\begin{array}{l}14.50 \\
\pm 2.95\end{array}$ & $\begin{array}{l}14.25 \\
\pm 2.34\end{array}$ \\
\hline & Tukey & a & $a$ & a & a & a & a & $a$ & a \\
\hline & Mínimo & 0.30 & 0.30 & 0.40 & 0.50 & 0.32 & 0.29 & 0.34 & 0.26 \\
\hline$\circ \underset{\alpha}{z}$ & Máximo & 1.00 & 1.00 & 1.10 & 1.75 & 2.29 & 1.48 & 1.30 & 1.81 \\
\hline 岁 & $\begin{array}{l}\text { Média } \\
\pm D P\end{array}$ & $\begin{array}{c}0.69 \\
\pm 0.21\end{array}$ & $\begin{array}{c}0.53 \\
\pm 0.19\end{array}$ & $\begin{array}{c}0.72 \\
\pm 0.20\end{array}$ & $\begin{array}{c}1.06 \\
\pm 0.41\end{array}$ & $\begin{array}{c}0.82 \\
\pm 0.47\end{array}$ & $\begin{array}{c}0.77 \\
\pm 0.37\end{array}$ & $\begin{array}{c}0.77 \\
\pm 0.29\end{array}$ & $\begin{array}{c}0.79 \\
\pm 0.33\end{array}$ \\
\hline & Tukey & $a$ & $a$ & a & $b$ & $a$ & a & a & $a$ \\
\hline
\end{tabular}



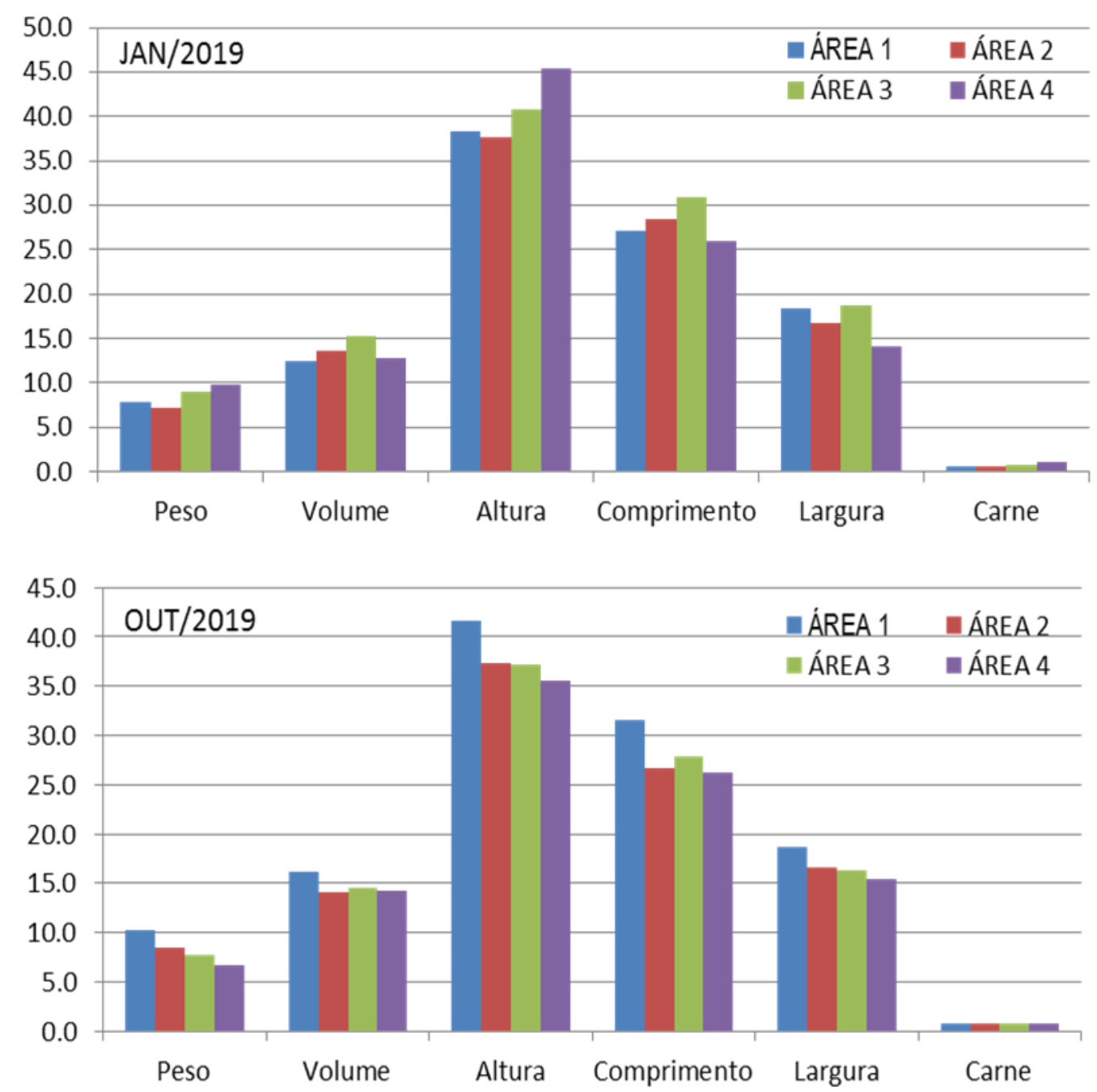

Figura 3. Peso $(\mathrm{g})$, volume $(\mathrm{ml})$, altura $(\mathrm{mm})$, comprimento $(\mathrm{mm})$ e largura $(\mathrm{mm})$ da ostra e peso úmido de carne ( $\mathrm{g}$ ) médios nas áreas A1 a A4 durante as amostragens nos períodos de (jan/2019) e de (out/2019).

O peso total das ostras variou entre 3.96 e $18.02 \mathrm{~g}$ na área $1 \mathrm{~A}$; entre 1.99 e $15.30 \mathrm{~g}$ na área A2 e entre 3.45 e $14.80 \mathrm{~g}$ e entre 2.39 e $18.70 \mathrm{~g}$, respectivamente nas áreas A3 e A4. Entre os períodos de coletas em Jan/2019 e Out/2019, verificou-se ganho médio de peso para as áreas $A 1$ e $A 2$ e perda para as áreas $A 3$ e $A 4$. Diferenças significativas $(p=0.0133)$ entre os pesos das ostras sendo detectado apenas entre as áreas A1 e A4 no período de outubro/2019.

O volume das ostras mostrou-se menos variável entre áreas e períodos de coleta. Nas áreas $\mathrm{A} 1$ e $\mathrm{A} 2$ o volume das ostras variou respectivamente entre 8 e $22 \mathrm{ml}$ e entre 4 e $27 \mathrm{ml}$ ao longo de todo o período do estudo, enquanto que nas áreas A3 e A4, apresentou valores de 8 a $18 \mathrm{~g}$ e de 4 a $22 \mathrm{~g}$. Não foram detectadas diferenças significativas entre os volumes medidos nas diferentes estações nem entre os períodos de coletas ou interação área x período.

As ostras do estuário do rio Ipojuca apresentaram alturas variáveis ao longo do estudo entre 30 e $49 \mathrm{~mm}$ na área $A 1$; entre 19 e $47 \mathrm{~mm}$ na área $A 2$, entre 24 e $48 \mathrm{~mm}$ na área $A 3$ e 
Santana and Medeiros, Características biométricas e índices de condição da ostra-de-mangue Crassostrea rhizophorae (Guilding, 1828) do estuário do rio Ipojuca, PE, Brasil.

entre 17 e $63 \mathrm{~mm}$ na área A4. As maiores variabilidades em altura dos indivíduos sendo registradas nas áreas $A 4$, mais interna e na área $A 2$, e a menor na área $A 1$ que recebe uma maior influência das águas marinhas. Durante o período de jan/2019 os indivíduos da área A4 apresentaram alturas estatisticamente maiores $\left(p=8.4 \times 10^{-6}\right)$ que os das demais áreas. Durante o período de out/2019, essa relação se inverteu, com indivíduos com alturas significativamente $(p=0.0102)$ maiores na área $A 1$ relativas aos das áreas $A 3$ e $A 4$.

Ao longo do estudo o comprimento e largura das ostras na área A1 variaram respectivamente entre 20 e $38 \mathrm{~mm}$ e entre 12 e $29 \mathrm{~mm}$. Na área A2, essa variação foi de 14 a $39 \mathrm{~mm}$ em comprimento e de 5 a $28 \mathrm{~mm}$ em largura. Os valores de comprimento e largura para a área A3 variaram entre 17 e $38 \mathrm{~mm}$ e entre 7 e $26 \mathrm{~mm}$, respectivamente. $\mathrm{Na}$ área $\mathrm{A} 4$, as ostras apresentaram comprimentos de concha de 10 a $38 \mathrm{~mm}$ e largura de concha variável entre 9 e $28 \mathrm{~mm}$. Diferenças significativas $(p=0.0020)$ foram detectadas entre as larguras das ostras das 4 estações amostrais, essas diferenças ocorrendo durante o período amostral de jan/2019 mas não em out/2019. A largura das ostras na área A4 sendo estatisticamente inferior àquelas das áreas A1 e A3. Em jan/2019 o comprimento médio das ostras na área A4, foi estatisticamente inferior àqueles da área $A 3$, sem diferenças significantes entre as demais estações de coleta. Já em out/2019 as ostras da área A1 apresentaram comprimentos significativamente superiores àqueles das ostras das áreas A2 e A4.

O peso médio de carne das ostras foi de $0.69 \mathrm{~g}(\min =0.30 \mathrm{~g} ; \max =1.00 \mathrm{~g})$ no período de jan/2019 e de $0.82 \mathrm{~g}(\min =0.32 \mathrm{~g} ; \max =2.29 \mathrm{~g})$ no período de out/2019 na área $A 1 ;$ de $0.54 \mathrm{~g}(\min =0.30 . \mathrm{g} ; \max =1.00 . \mathrm{g})$ no período de jan $/ 2019$ e de $0.77 \mathrm{~g}(\min =0.29 \mathrm{~g} ; \max =1.48$ g) no período de out/2019 na área $A 2$. Na área A3, o peso médio de carne das ostras foi de $0.72 \mathrm{~g}(\min =0.40 \mathrm{~g} ; \max =1.10 \mathrm{~g})$ no período de $j a n / 2019$ e de $0.77 \mathrm{~g}(\min =0.34 \mathrm{~g} ; \max =1.30$ g) no período de out/2019. Na área $\mathrm{A} 4$, as ostras apresentaram um peso médio de carne de $1.06 \mathrm{~g}(\min =0.50 \mathrm{~g} ; \max =1.75 \mathrm{~g})$ nas coletas em $(j a n / 2019)$ e de $0.79 \mathrm{~g}$ ( $\min =0.60$; $\max =1.81 \mathrm{~g}$ ) nas coletas em out/2019. O teste de ANOVA detectou diferenças significativas entre as áreas amostrais $(\mathrm{p}=0.0021)$ e para a interação área amostral $\mathrm{x}$ período de coleta $(p=0.0031) . E m$ jan/2019 o peso da carne na área A4 significativamente superior àqueles das demais áreas, sem diferenças significativas entre as 4 áreas em out/2019.

$\mathrm{Na}$ Fig. 4, são apresentadas as relações altura $\mathrm{x}$ peso para as ostras das 4 áreas amostradas em Jan/2019 e Out/2019. Em Jan/2019 as correlações entre essas variáveis $(n=20)$ foram altamente significativas para as áreas $A 1\left(r^{2}=0.98\right) ; A 2\left(r^{2}=0.95\right), A 3\left(r^{2}=0.96\right)$ e A4 $\left(r^{2}=0.69\right)$ ainda que os valores nesta última área apresentassem uma maior dispersão. $A$ mesma tendência foi verificada em Out/2019, com correlações altamente significantes para as áreas $A 1, A 2, A 3$ e $A 4\left(r^{2}=0.93 ; r^{2}=0.91, r^{2}=0.85\right.$ e $r^{2}=0.80$, respectivamente $)$. 

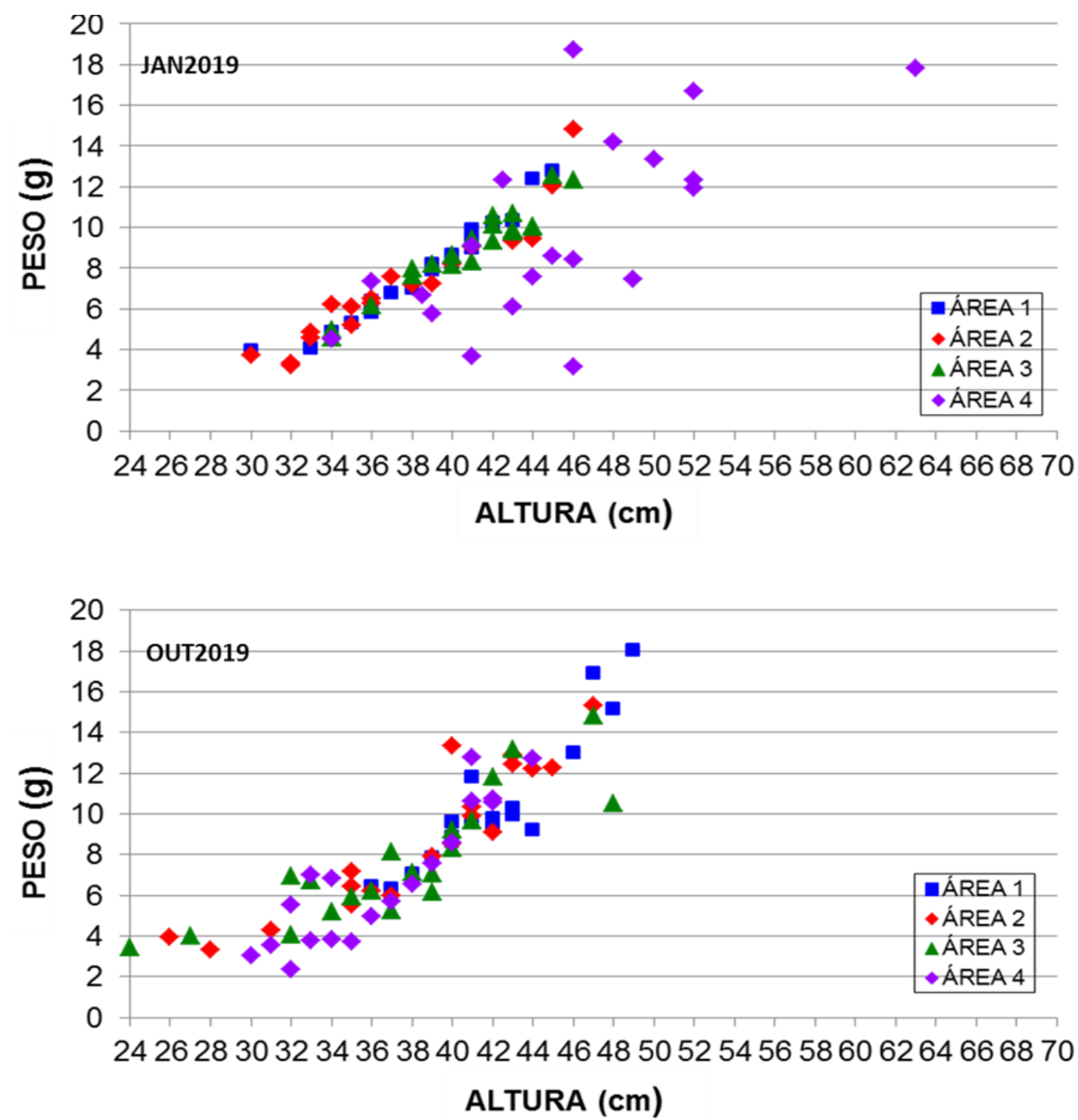

Figura 4. Correlação de altura (mm) x peso (g) nas áreas A1 a A4 em jan/2019 e out/2019.

\section{ÍNDICES DE MONITORAÇÃO}

Nas Tab. 3 e Fig. 5 são apresentados os índices de STI1 e AFNOR de ostras-de-mangue do rio Ipojuca para os períodos de jan/2019 e de out/2019.

Valores do índice STI1 para a área de estudo variaram entre 2.38 e 2.62 em jan/2019 e entre 2.58 e 3.53 em out/2019. Diferenças significativas foram detectadas entre as amostragens em jan/2019 e out/2019 para as áreas amostrais A2, A3 e A4, com os valores mais elevados detectados em out/2019. Não foram verificadas diferenças significativas deste índice entre as quatro áreas amostrais em ambos períodos de coleta.

Valores do índice AFNOR apresentaram grande variabilidade entre as 4 estações amostrais, variando de 0.08 a 8.91 em jan/2019 e entre 7.71 e 13.18 em out/2019. Diferenças significantes entre os valores desse índice determinados em jan/2019 e out/2019 foram 
Santana and Medeiros, Características biométricas e índices de condição da ostra-de-mangue Crassostrea rhizophorae (Guilding, 1828) do estuário do rio Ipojuca, PE, Brasil.

verificados para as áreas amostrais A1, A2 e A3. Em ambos os períodos amostrais, o valor do índice AFNOR foi significativamente mais elevado à área amostral A4 relativo às demais.

Tabela 3. Valores dos índices STI1 [-] e AFNOR (\%) das ostras do estuário do rio Ipojuca (áreas A1 a A4) em Jan/2019 e out/2019.

\begin{tabular}{cccc}
\hline Período & Área & STI1 & AFNOR \\
\hline \multirow{3}{*}{ JAN/2019 } & A1 & 2.51 & 8.91 \\
& A2 & 2.50 & 7.53 \\
& A3 & 2.38 & 0.08 \\
AUT/2019 & A4 & 2.62 & 0.13 \\
& A1 & 2.58 & 7.71 \\
& A2 & 3.41 & 9.29 \\
& A3 & 2.96 & 10.22 \\
& A4 & 3.53 & 13.18 \\
\hline
\end{tabular}
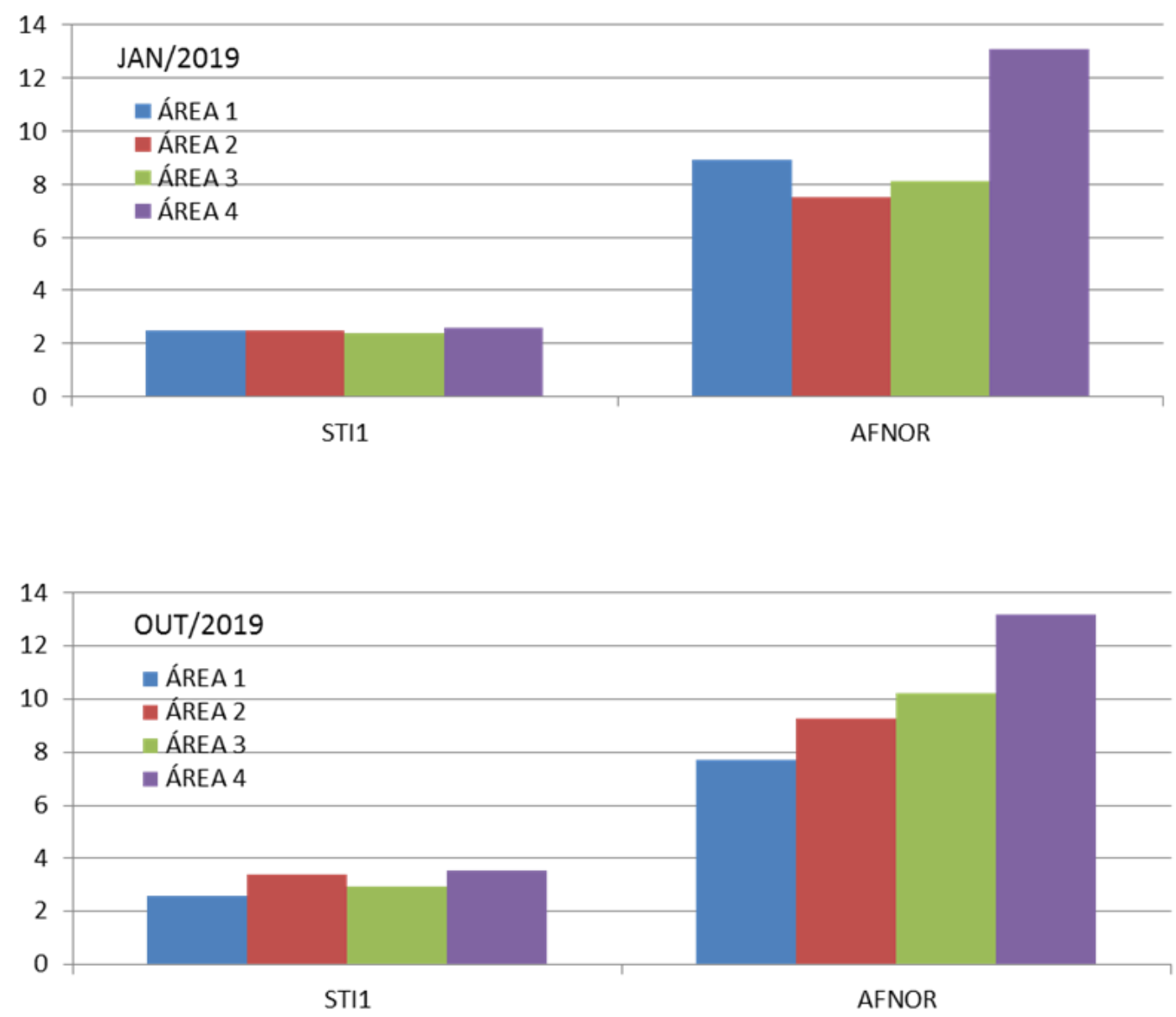

Figura 5. Índices de Condição STI1 (1-10) e AFNOR (0 - $\%)$ das ostras nas áreas A1 a A4 do rio Ipojuca em jan/2019 e out/2019. 


\section{DISCUSSÃO}

O reconhecimento da importância dos sistemas naturais e de sua acelerada degradação tem impulsionado mais e mais ações voltadas para sua revitalização e recuperação. De acordo com o levantamento "Indicadores de Desenvolvimento Sustentável", do Instituto Brasileiro de Geografia e Estatística (IBGE), os rios brasileiros estão aumentando o seu nível de poluição. Os estuários, embora reconhecidamente constituam ambientes que devam ser poupados da degradação ambiental, acham-se grandemente comprometidos pelas alterações decorrentes da atividade humana direta ou remota (Miranda et al., 2002). Este é também o caso do estuário do rio Ipojuca no estado de Pernambuco cujo Índice de Qualidade da Água (IQA) calculado pelo IBGE foi o terceiro pior dos rios mais poluídos do país (IBGE, 2015).

A recuperação dos sistemas estuarinos inicia-se pela revitalização dos bancos de ostras nos mesmos (Harding and Mann, 2001; Neema, 2008) uma vez que eles contribuem para a melhoria da qualidade da água dos sistemas estuarinos (Dame, 2011). Como filtradores as ostras removem microalgas, material orgânica em suspensão e possivelmente dissolvida da coluna d'água retendo-os em suas brânquias para se alimentarem (Peterson et al., 2003) melhorando sua qualidade e favorecendo a recuperação de vários outros organismos aquáticos, sobretudo dos crustáceos e peixes.

Vários fatores de origem físicas (Souza et al., 2017; Wolanski, 2008) e biológicas e químicas (Poli, 2004), podem influenciar na distribuição e no desenvolvimento de ostras em um manguezal.

Segundo Guimarães et al. (2008), em vários manguezais do NE-brasileiro salinidades de 8 a 14 tem se mostrado mais propícias para o desenvolvimento das ostras-de-mangue, embora as ostras sejam bem tolerantes a uma larga faixa de salinidade. Isto explicaria a maior concentração de ostras na área de estudo e sua ausência no alto estuário e próximo à sua desembocadura onde as salinidades médias correspondem respectivamente a 5 e 35 (Lins and Medeiros, 2018). Ainda, mesmo dentro da faixa ideal existe uma relação entre a salinidade e a taxa de reprodução das ostras-de-mangue. Em geral, quanto mais alta a salinidade (até o seu limite de 34) maior é a taxa de reprodução desses indivíduos (Vilanova and Chaves, 1988).

O rio Ipojuca recebe maior descarga fluvial de março a agosto, principalmente de maio a julho e menor descarga de setembro a fevereiro, principalmente de dezembro a janeiro (Medeiros and Lins, 2018). No estuário do rio Ipojuca, o período de desova da ostra-demangue, ocorre geralmente durante os meses mais quentes (Costa, 1975; SEBRAE, 2005) iniciando por volta do mês de outubro, na transição do período chuvoso para o de estiagem. O início do período reprodutivo das ostras naturalmente proporciona um aumento gradual da massa desses animais (Galtsoff, 1964). O aumento de peso total da ostra, e de peso e volume de carne, por exemplo, na verdade evidencia gônadas maiores com gametas maduros, 
Santana and Medeiros, Características biométricas e índices de condição da ostra-de-mangue Crassostrea rhizophorae (Guilding, 1828) do estuário do rio Ipojuca, PE, Brasil.

enquanto a redução nestes valores pode estar associada a gônadas vazias (Nascimento and Pereira, 1980). Tais relações podem elucidar os resultados do presente trabalho, que indicaram uma tendência a ganho de peso de carne para as ostras das áreas $A 1, A 2$ e A3 e A2 entre as coletas de jan/2019 e de out/2019 e uma perda de peso para a área A4.

A desova das ostras é condicionada pela expectativa de ambiente favorável para o crescimento de suas larvas. Por ambiente favorável, se traduz condições de temperatura e salinidade, que propiciem bom crescimento e sobrevivência, como também de presença de alimento (Mann and Steinke, 1988; Roegner et al., 1990) para as larvas, o que não necessariamente ocorre simultaneamente em todas as regiões de um estuário como relatado para a espécie C. gigas e C. gasar (Paixão et al., 2013) e para a espécie em questão, $C$. rhizophorae (Lenz and Bohers, 2011). A perda de peso de carne na estação A4 contra ganho de peso de carne nas demais estações em out/2019 relativa a jan/2019 pode ser indicativa de que a desova na estação A4 ocorreu mais cedo que nas demais estações. Levantamentos realizados por Santana (2019) no mesmo período e estações amostrais evidenciaram um maior número de fixação de sementes de ostras na estação A4 em out/2019, reforçando esta hipótese.

Segundo Alzieu et al. (1989) as ostras podem apresentar malformação e/ou um espessamento de suas conchas quando em presença de TBT (Tributil-de-Estanho). A alteração morfológica da concha para uma forma mais esférica (balling) é um dos efeitos crónicos mais relevantes e facilmente identificáveis da exposição de moluscos a este composto. O TBT é um produto utilizado na composição de pinturas para proteção dos cascos das embarcações contra bioincrustantes, embora sua utilização tenha sido restringida no Brasil desde 2010. Além de ser empregado em pinturas antiincrustantes, ele é também utilizado pela indústria de plásticos e na agricultura. Na indústria de plástico, como agente estabilizador da degradação do Polivinilo de Cloreto (PVC) pelo efeito da luz e/ou calor e como flexibilizante, para conferir uma maior flexibilidade aos plásticos. Na agricultura como pesticida, conservante de madeiras, e em menor escala, como desinfectantes e em tratamentos algicidas em materiais de construção (Borges, 1997). Valores do índice STI1 de 10 indicam ausência de contaminação e valores próximo a 1 contaminação muito elevada.

As ostras amostradas ao longo do estuário do rio Ipojuca nas áreas $A 1, A 2, A 3$ e $A 4$ apresentaram valores de STI1 entre 2.38 e 3.53 (contaminação elevada) indicativos de uma possível exposição das ostras a esse composto. Os maiores índices de contaminação (menores valores do índice STI1) foram detectados em jan2019 nas 4 áreas amostrais A1 a A4 durante a estação de estiagem. Já em out2019, ao final da estação chuvosa, verificou-se um gradiente com menores valores da estação $A 1$ mais externa em direção à estação $A 4$, mais interna ao estuário. Novos estudos incluindo determinações de TBT nos sedimentos e em ostras são necessários para elucidar essas questões. 
$O$ índice de qualidade AFNOR, (Soletchnik et al., 2000), varia de acordo com a pressão sofrida pela ostra durante o seu crescimento. Segundo este índice, as ostras da área A4 seriam classificadas como "especiais" tanto em jan/2019 quanto out/2019. Já as ostras das áreas A2, A3 e A4 tiveram seus maiores índices nas coletas em out/2019, quando seriam classificadas como "finas", enquanto que na área A1 este índice foi mais elevado nas coletas de jan/2019. Em jan/2019 as ostras das áreas A1, A2 e A3 são classificadas como "finas". Porém as da área A3 puderam ser classificadas como especiais em out/2019. Nenhuma ostra apresentou índice menor que 6,5 (mínimo). Além de ser um índice de importante relevância comercial (Magro, 2016), tal proporção reflete a condição do ambiente, seja ele controlado ou não. O estuário do Ipojuca possui condições de aporte nutricional para o desenvolvimento da espécie em questão, visto os valores obtidos, mesmo tendo sido considerado um dos três rios mais polidos do pais (IBGE, 2015).

\section{CONCLUSÃO}

A biometria de ostras coletadas de vários pontos do estuário do rio Ipojuca e a aplicação de índices de monitoramento revelaram por um lado a ocorrência de ostras que comercialmente seriam classificadas como "finas" e mesmo "especiais" em termos de seu desenvolvimento e preenchimento do espaço intervalvar das mesmas (índice AFNOR sempre superior a 6,5). Por outro lado, baixos valores do índice de monitoramento STI1 (inferiores a 4), forte indicador de contaminação por tributil-estanho TBT, alertam para a necessidade de futuras investigações para confirmar essas suspeitas bem como para investigar a adequabilidade ou não das ostras do Ipojuca ao consumo humano.

\section{AGRADECIMENTOS}

Os autores agradecem ao Sr. Isaac Freitas e ao Sr. Josimar Vicente de Araújo pelo apoio durante os trabalhos de mar.

\section{REFERÊNCIAS}

AFNOR (1985), Norme française huîtres creuses, Dénomination et classification, NF, Vol. 45-56, $5 p$.

Agência Estadual de Planejamento e Pesquisas de Pernambuco (CONDEPE/FIDEM) (2005), Série Bacias Hidrográficas de Pernambuco, Vol. 1, Rio Ipojuca, pp. 1-64.

Alzieu, C., Heral, M., Thibaud, Y., Dardignac, M.J. and Feuillet, M. (1982), Influence des peintures antissalissures à base d' organostanniques sur la calcification de la coquille de 
Santana and Medeiros, Características biométricas e índices de condição da ostra-de-mangue Crassostrea rhizophorae (Guilding, 1828) do estuário do rio Ipojuca, PE, Brasil.

l'ûitre Crassostrea gigas, Revue des Travaux de l'Institut des Pêches Maritimes, Vol. 45, pp. $100-116$.

Andrade, G.O. and Lins, R.C.O. (2001), Clima do Nordeste, Revista de Geografia, DCG/UFPE NAPA, Recife, Vol. 17, n. 1, pp. 3-32.

Borges, L.M.S. (1997). Alguns aspectos da biologia de Thais haemastoma (Gastropoda: Muricidae) e a sua utilização como espécie indicadora de poluição por TBT, nas águas do porto e marina de Ponta Delgada. Estágio de licenciatura em biologia, Universidade dos Açores, Ponta Delgada. 133 pp.

Braga, R.A.P., Maestrini, P. and Lins, M.F. (1989), Impacto da implantação do Complexo Industrial-Portuário de Suape - PE sobre as populações de moluscos comestíveis, Anais da Sociedade Nordestina de Zoologia (Teresina-Piauí-Brasil), Vol. 3, n. 3, pp.137-153.

Comissão Interministerial para os Recursos do Mar (CIRM) (1981), III Plano Básico de Desenvolvimento Científico e Tecnológico, in I Plano Setorial para os Recursos do Mar (1982-1985), Brasília, pp. 1-105.

CÓDIGO FLORESTAL - Lei No 12.651 de 25 de maio de 2012 (2012), Dispõe sobre a proteção da vegetação nativa e dá outras providencias, DOU de 28 de maio de 2012.

Costa, P.F. (1975), Biologia e tecnologia para o cultivo de ostras, in Marinha do Brasil (MB), Instituto Nacional de Estudos do Mar, Manual de Maricultura, Rio de Janeiro, cap. VIII, $36 p$.

Dame, R.F. (2011), Ecology of Marine Bivalves: An Ecosystem Approach, CRC Press, Boca Raton, FL. 284p.

Galtsoff, P.S. (1964), The American oyster Crassostrea virginica (Gmelin), Fishery Bulletin, Vol. 64, n. 1, pp. 11-28.

Gervis, M.H. and Sims, N.A. (1992), The biology and culture of pearl oysters (Bivalvia: Pteriidae), ICLARM Studies and Reviews, 21, Overseas Development Administration, UK and ICLARM, Manila.

Grabowski, J.H. and Petersen, C.H. (2007), Restoring Oyster Reefs to Recover Ecossystem Services, Theoretical Ecology Series, Vol. 4, pp. 281-298. DOI: 10.1016/S1875306X(07)80017-7

Guimarães, I.M., Antonio, I.G., Peixoto, S. and Olivera, A. (2008), Influência da salinidade sobre a sobrevivência da ostra-do-mangue, Crassostrea rhizophorae, Arquivos de Ciências do Mar, Fortaleza, Vol. 41, n.1, p.118-122. DOI: 10.32360/acmar.v41i1.6084

Harding, J.M. and Mann, R. (2001), Oyster reefs as fish habitat: opportunistic use of restored reefs by transient fishes, Journal of Shellfish Research, Vol. 20, pp. 951-959. 
Hargis Jr., W.J. and Haven, D.S. (1999), Chesapeake oyster reefs, their importance, destruction and guidelines for restoring them, in Luckenbach, M.W., Mann, R. and Wesson, J.A. (Eds), Oyster reef habitat restoration: a synopsis of approaches, Virginia Inst Mar Sci Press, Gloucester Point, VA, pp. 329-358.

Instituto Brasileiro de Geografia e Estatística (IBGE) (2015), Indicadores de Desenvolvimento Sustentável - Brasil, 352p.

Jackson, J.B.C., Kirby, M.X., Berger, W.H, Bjorndal, K.A., Botsford, L.W., Bourque, B.J., Bradbury, R.H., Cooke, R., Erlandson, J., Estes, J.A., Hughes, T.P., Kidwell, S., Lange, C.B., Lenihan, H.S., Pandolfi, J.M., Peterson, C.H., Steneck, R.S., Tegner, M.J. and Warner, R.R. (2001), Historical overfishing and the recent collapse of coastal ecosystems, Science, Vol. 293, pp. 629-638. DOI:10.1126/science.1059199.

Lenz, T. and Boehs, G. (2011), Ciclo reprodutivo del ostión de manglar Crassostrea rhizophorae (Bivalvia: Ostreidae) en la Bahía de Camamu Bahia, Brasil, Revista de Biologia Tropical, Vol. 59, n. 1, pp. 137-149. DOI: 10.15517/rbt.v59i1.3184.

Lins, S.R.R.M. and Medeiros, C. (2018), Propagação da maré salina em um estuário tropical estrangulado, Ipojuca, NE-Brasil, Tropical Oceanography, Vol. 46, n. 1, pp. 70-91. DOI: https://doi.org/10.5914/tropocean.v46i1.237251.

Magro, C.A.L. (2016), Avaliação preliminar da Qualidade, Segurança e Perfil Nutricional da fração edível de Crassostrea spp. selvagem e de aquacultura no Sado, Dissertação de Mestrado em Tecnologias de Produção e Transformação, Universidade Federal do Pará, 69p.

Mann, R. (1982), Ecology of Coastal Waters: a System Approach, University of California Press, Verkeley, CA, 322p.

Mann, F.D. and Steinke, T.D. (1988), Photosynthetic and respiratory responses of the mangrove-associated red algae, Bostrychia radicans e Caloglossa leprieurii, South African Journal of Botany, Vol. 54, pp. 203-207. DOI: https://doi.org/10.1016/S0254$\underline{6299(16) 31320-5}$

Martinez, R.H. and Rusch, E. (2014), Understanding the connection between coastal waters and ocean ecosystem series and human health, Workshop Summary 2014, Institute of Medicine of National Academy - versão eletrônica, National Academic Press, available at: http://www.nap.edu/catalog.php?record_id=18552.

Miranda, L.B., Castro, B.M. and Kjerfve, B. (2002), Princípios de Oceanografia Física de Estuários, Ed. da Universidade de São Paulo, 424p. 
Nascimento, I.A. and Pereira, S.A. (1980), Change in the condition index from mangrove oysters (Crassostrea rhizophorae) from Todos os Santos Bay, Salvador, Brazil, Aquaculture, Vol. 20, pp. 9-15. DOI: https://doi.org/10.1016/0044-8486(80)90057-5.

Neumann-Leitão, S. (1994), Impactos antrópicos na comunidade zooplanctônica estuarina. Porto de Suape - PE - Brasil, Tese de Doutorado em Ciências da Engenharia Ambiental, Departamento de Hidráulica, Escola de Engenharia de São Carlos, Universidade de São Paulo, 273p. DOI: https://doi.org/10.1590/S1516-89132003000100012.

Newell, R.I.E., Cornwell, J.C. and Owens, M.S. (2002), Influence of simulated bivalve biodeposition and microphytobenthos on sediment nitrogen dynamics: A laboratory study, Limnology and Oceanography, Vol. 47, n. 5, pp. 1367-1379. DOI: https://doi.org/10.4319/lo.2002.47.5.1367.

Núcleo de Estudos em Economia do Meio Ambiente (NEEMA) (2008), Difusão de Tecnologias Sustentáveis, Inovações para o Desenvolvimento, Ostreicultura, em: http://www.neema.ufc.br.

Paixão, L., Ferreira, M.A., Nunes, Z., Fonseca-Sizo, F. and Rocha, R. (2013), Effects of salinity and rainfall on the reproductive biology of the mangrove oyster (Crassostrea gasar): Implications for the collection of broodstock oysters, Aquaculture, Vol. 380-383, pp. 6-12. DOI: https://doi.org/10.1016/j.aquaculture.2012.11.019.

Peterson, C.H., Grabowski, J.H. and Powers, S.P. (2003), Estimated enhancement of fish production resulting from restoring oyster reef habitat: quantitative variation, Marine Ecology Progress Series, Vol. 264, pp. 249-264. DOI: 10.3354/meps264249.

Poli, C.R. (2004), Cultivo de ostras do pacífico (Crassostrea gigas, 1852), in Poli, C.R., Poli, A.T., Andreatta, E. and Beltrame, E. (Orgs.), Aqüicultura: Experiências Brasileiras, UFSC Multitarefa Editora LTDA., pp. 251-266.

Roegner, G., Curtis, A. and Mann, R. (1990), Settlement Patterns of Crassostrea virginica (Gmelin) Larvae in Relation to Tidal Zonation, VIMS Articles, 1283, em: https://scholarworks.wm.edu/vimsarticles/1283.

Santana, L.L.S. (2019), Hidrodinâmica Estuarina: interação com a arquitetura das raízes de Rhizophora mangle (Nascimento 1983) e implicações para a dispersão e fixação de larvas de Crassostrea rhizophorae (Guilding 1828) no rio Ipojuca, Pernambuco, Brasil, Dissertação de Mestrado em Oceanografia, Departamento de Oceanografia, Universidade Federal de Pernambuco.

Serviço Brasileiro de Apoio às Micro e Pequenas Empresas (SEBRAE) (2005), Ostreicultura manual de boas práticas: qualidade e segurança para bons negócios, 55 p. 
Soletchnik, P., Le Moine, O., Goulletquer, P., Geairon, P., Razeta, D., Faury, N., Fouché, D. and Robert, S. (2000), Optimisation of the traditional Pacific cupped oyster (Crassostrea gigas Thunberg) culture on the French Atlantic coastline: autumnal fattening in semi-closed ponds, Aquaculture, Vol. 199, pp. 73-91. DOI: https://doi.org/10.1016/S00448486(01)00554-3.

Souza, T.R., Moreira, L.P., Andrade, L., Ramires, M., Sadauskas-Henrique, H. and Barrella, W. (2017), Análise do tamanho das ostras de mangue (Crassostrea rhyzophorae) em diferentes ambientes do manguezal (remanso e correnteza) na Reserva de Desenvolvimento Sustentável Barra do Una, Peruíbe-SP, Anais do Encontro Nacional de Pós-Graduação, VI ENPG, Vol. 1, pp. 276-279.

Vilanova, M.F.V. and Chaves, E.M.B. (1988), Contribuição para o conhecimento da viabilidade do cultivo de ostra-do-mangue Crassostrea rhizophorae (Guilding, 1828) (Mollusca: Bivalvia), no estuário do Rio Ceará, Ceará, Brasil, Arquivos de Ciências do Mar, Vol. 27, pp. 111-125.

Wolanski, E. (2008), Outline of the physical processes within mangrove systems and its implications for biodiversity, London: CRC Press. 